\title{
Kinematics of pantograph masts
}

\author{
B. P. Nagaraj, R. Pandiyan ${ }^{\dagger}$ \\ ISRO Satellite Centre, \\ Bangalore, 560 017, India \\ and \\ Ashitava Ghosal ${ }^{\ddagger}$ \\ Dept. of Mechanical Engineering, \\ Indian Institute of Science, \\ Bangalore 560 012, India.
}

\begin{abstract}
This paper deals with the kinematics of pantograph masts. Pantograph masts have widespread use in space application as deployable structures. They are over constrained mechanisms with degree-of-freedom, evaluated by the Grübler-Kutzbach formula, as less than one. In this paper, a numerical algorithm is used to evaluate the degreeof-freedom of pantograph masts by obtaining the null-space of a constraint Jacobian matrix. In the process redundant joints in the masts are obtained. A method based on symbolic computation, to obtain the closed-form kinematics equations of triangular and box shaped pantograph masts, is presented. In the process, the various configurations such masts can attain during deployment, are obtained. The closed-form solution also helps in identifying the redundant joints in the masts. The symbolic computations involving the Jacobian matrix also leads to a method to evaluate the global degree-offreedom for these masts.
\end{abstract}

Keywords: Deployable masts, Degree-of-freedom, Jacobian matrix, Closed-form solutions

\section{Introduction}

Deployable structures is a generic name for a broad category of prefabricated structures that can be transformed from a closed compact configuration to a predetermined expanded form in which they are stable and can carry loads. The study of deployable/collapsible mast has

\footnotetext{
*Spacecraft Mechanisms Group

$\dagger$ Flight Dynamics Division

${ }^{\ddagger}$ Corresponding author. email: asitava@mecheng.iisc.ernet.in
} 
applications in space industry that requires a highly collapsible and portable mechanism to be carried in a spacecraft and expandable in space for payloads. Deployable/foldable mast have one or more so-called internal mechanisms $[1,2]$ and their degree-of-freedom as evaluated by the Grübler-Kutzbach criterion often turns out to be less than one. In this paper, the kinematics of deployable masts made up of pantograph mechanisms or scissor like element(SLE) is studied. An SLE in two dimensional form has straight rods of equal length connected by pivots in the middle. The assembly has one degree-of-freedom and the basic model can be folded and deployed freely. Three dimensional masts are created with SLE in such a way that the SLE's form a structural unit which in plan view is a normal polygon with each side being an SLE. The polygon can be equilateral triangle, square or normal $n$-sided polygon. By combining several of these normal polygon shaped units, structures of various geometric configurations can be created. Active cables control the deployment and pre-stress the pantograph and passive cables are pre-tensioned in the fully deployed configuration. These cables have the function of increasing the stiffness when fully deployed [3]. The whole system deploys synchronously.

The kinematics of pantograph masts can be studied by use of relative coordinates [4], reference point coordinates (as used in the commercial software ADAMS) or Cartesian coordinates [5]. In this paper Cartesian coordinates, also called natural or basic coordinates, have been used. This method uses the constant distance condition for two or more basic points of the same link and constant area of triangle for the prismatic pair [6]. Using unitary vectors the method can be extended to spatial mechanisms [7]. The main advantage of using Cartesian coordinates is that the constraint equations are quadratic as opposed to transcendental equations for relative coordinates, and the number of variables tends to be (on average) in between relative coordinates and reference point coordinates. In the analysis of SLE's, previous researchers [8] have formulated the foldability equation for translational, polar and Hoberman's units formed by SLE based on a geometric approach. This approach is extended to combination of above units for triangular and box shaped structures. The equations of motion for the stacked SLE masts were obtained and solved numerically using Cartesian coordinates [9]. They assumed the joints connecting SLEs as spherical. To the best of our knowledge, closed-form kinematics equations for these masts have not been attempted by researchers. Closed-form equations, when available, are expected to considerably reduce computation time. They will also allow us to obtain different configurations a mast can attain and helps in better design of the deployable structures. In this paper the closed form kinematics equations are derived for the triangular and box mast using the symbolic manipulation software Mathematica [10].

Typical deployable masts have large number of links and joints. The degree-of-freedom of these masts, as evaluated by the Grübler-Kutzback criteria, often gives numbers less than one and hence, the degree-of-freedom formula does not give a true number. Another known deployable mechanism with SLE's is the articulated ball mechanism with multiple loops described in [11]. The authors in [11] have used screw theory to evaluate the mobility of this ball mechanism. For metamorphic mechanisms, such as a Chinese lantern and a hexahedron, the authors [12] have attempted to obtain the order of the screw for use in the 
Grübler-Kutzback criteria. More recently, a new method for evaluating the degree-of-freedom of a mechanism based on the output members rather than the whole mechanism is presented in [13]. This approach is also based on screw theory and has been applied to obtain correct degree-of-freedom for a closed-loop parallel mechanism. Gogu [14] presents an exhaustive literature survey of methods to obtain mobility of mechanisms. The author also presents a formula, obtained using symbolic manipulation software, for calculating the mobility of closed loop parallel mechanism, with at least two elementary legs, by evaluating the rank of loop closure equations. A related approach is the use of graph theory for the topological synthesis of deployable mechanism [15]. For a deployable mechanism, an equivalent graph with edges (joints) and nodes (links) is generated. Warner and Chew [16] have presented a procedure that enables exhaustive generation of these graphs for given number of nodes and edges. The same authors have extended this procedure to the truss modules which are commonly used as building blocks for large structures [17]. Graph theory based approaches normally do not take into account the dimensions of the links. However, the authors in [17] claim that their procedure generates information on the relative lengths of the links in the truss modules by examining the graphs. The concept of using a Jacobian matrix to evaluate the mobility was first presented by Freudenstein [18] for an over-constrained mechanism. Later, the first and higher order derivatives of constraint equations has been used for under constrained structural systems to evaluate mobility and state of self stress by Kuznetsov [19]. In this paper, similar to the approach of Garcia and Bayo [5], the natural coordinates and the derivatives of the constraint equations are used to obtain the correct degrees-of-freedom of deployable masts. A numerical algorithm similar to the one developed by Garcia and Bayo [5] is used to identify redundant joints/links in a mast which leads to incorrect degreesof-freedom from the Grübler-Kutzback criteria. A new method based on the constraint Jacobian matrix to evaluate the global degree-of-freedom of the mast is presented.

This paper is organized as follows: In section 2, a brief description of the deployable masts considered for the analysis is presented. Section 3 presents the mathematical approach for the evaluation of degrees-of-freedom for spatial deployable mast followed by an algorithm to obtain the redundant joints in masts. In section 4, the detailed formulation to evaluate the closed-form kinematic solution for the triangular and box mast is presented. In section 5, a method to evaluate the global degree-of-freedom of the mast from the Jacobian matrix is presented. Section 6 illustrates our approach by finding redundant joints and also present a simulation of a triangular mast. Finally, conclusions are presented in Section 7.

\section{Kinematic description of the masts}

As mentioned earlier, most masts have an SLE as its basic unit. The simplest planar SLE is shown in Figure 1. The revolute joint in the middle connect the two links of equal length. The assembly has one degree-of-freedom and the SLE remains stress free during the folding and extending process.

The triangular mast and box mast, built out of SLE's, are presented in Figure 2 and Figure 3 respectively. The triangular mast has three SLE's and the box mast has four 


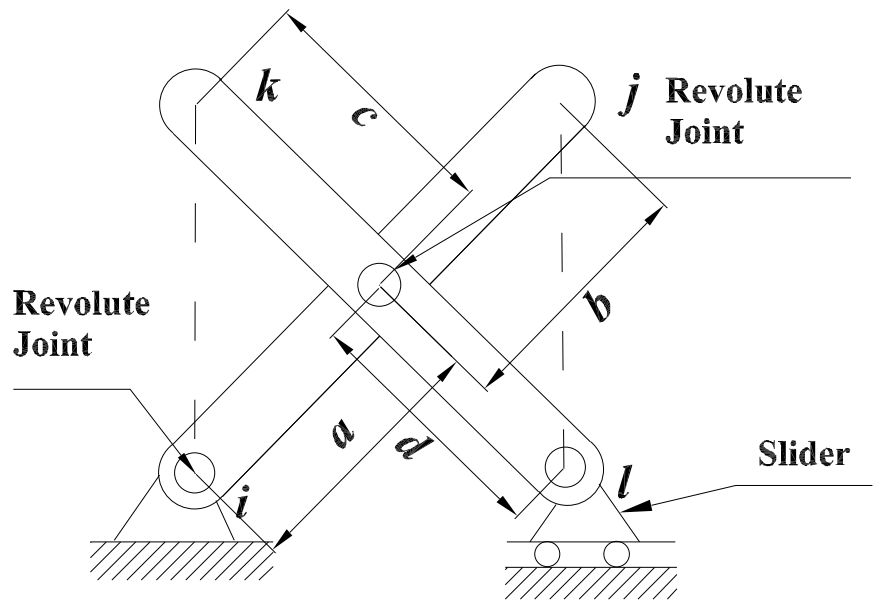

Figure 1: Basic module of SLE

SLE's. The masts are deployed using active cables connected to a motor which is usually attached to a fixed base. Many space deployable structures have several triangular or boxed masts stacked one on top of the other. In the figures the joints connecting the SLE's are shown to be spherical joints. However, in actual masts the spherical joints are replaced with revolute joints.

\section{$3 \quad$ Kinematic modeling}

The natural coordinates [5] to model masts are used. When using natural coordinates, the links must contain sufficient number of points and unit vectors so that their motion is completely defined. A point is located on those joints in which there is a common point to the two links. A unit vector must be positioned on joints that have rotation or translational axis. All points of interest whose position are to be considered as a primary unknown variable are defined as basic points. In the natural coordinate system the constraint equations originate in the form of rigid constraints of links and joint constraints. 


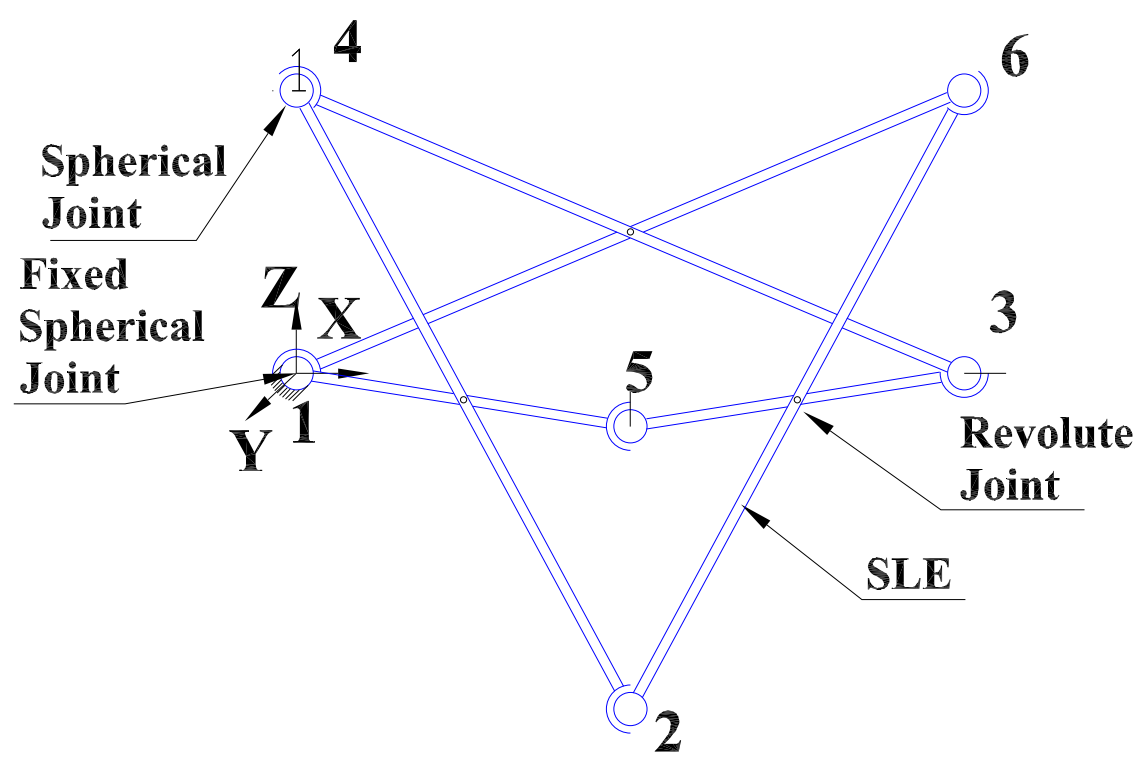

Figure 2: Triangular SLE mast

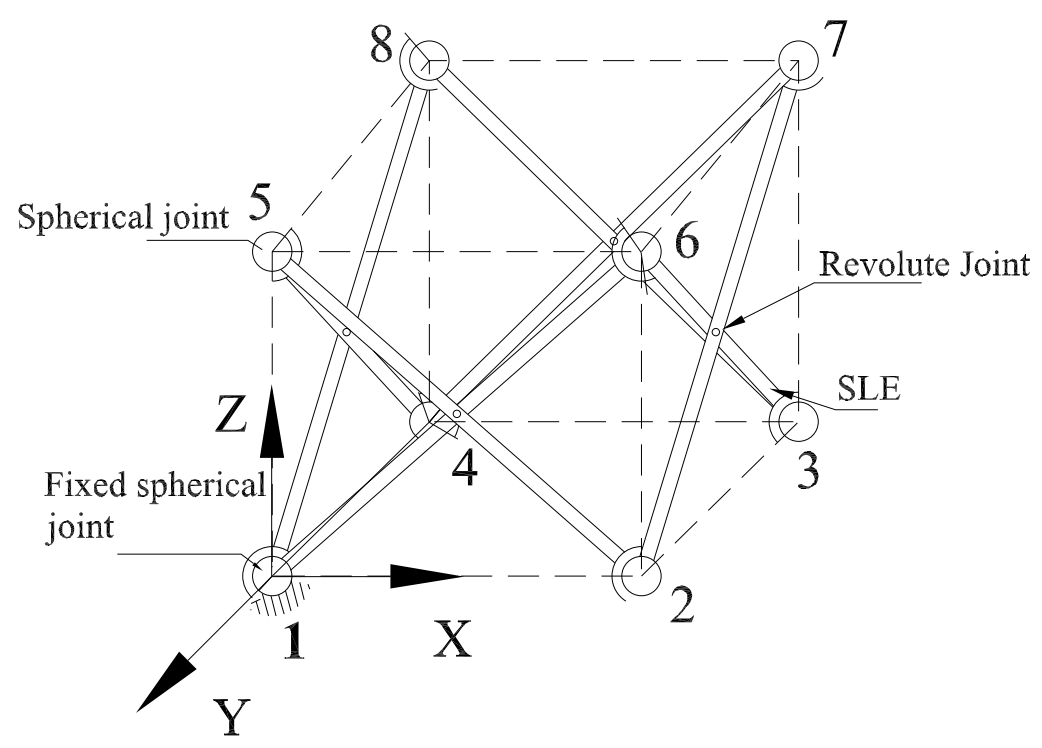

Figure 3: Box SLE mast 


\subsection{Rigid link constraint}

A rigid link is characterized by a constant distance between two natural coordinates $i$ and $j$. This is given by

$$
\mathbf{r}_{i j} \cdot \mathbf{r}_{i j}=L_{i j}^{2}
$$

where $\mathbf{r}_{i j}=\left[\left(X_{i}-X_{j}\right),\left(Y_{i}-Y_{j}\right),\left(Z_{i}-Z_{j}\right)\right]^{T}$ with $\left(X_{i}, Y_{i}, Z_{i}\right),\left(X_{j}, Y_{j}, Z_{j}\right)$ are the natural coordinates of $i, j$, respectively, and $L_{i j}$ is the distance between $i$ and $j$.

\subsection{Joint constraints}

Joint constraints are related to the relative motion between the links connected by the joint. The constraints corresponding to spherical joint is automatically satisfied when adjacent links share a basic point. Revolute joint is formed when two adjacent links share a basic point and an unit vector. The link shown in Figure 4 has two basic points $i$ and $j$ and two non-coplanar unit vectors $\mathbf{U}_{\mathbf{m}}$ and $\mathbf{U}_{\mathbf{n}}$ with angles $\alpha$ and $\beta$ with the link. The constraint equations for a revolute joint are

$$
\begin{array}{r}
\mathbf{r}_{i j} \cdot \mathbf{U}_{m}-L_{i j} \cos (\alpha)=0 \\
\mathbf{r}_{i j} \cdot \mathbf{U}_{n}-L_{i j} \cos (\beta)=0
\end{array}
$$

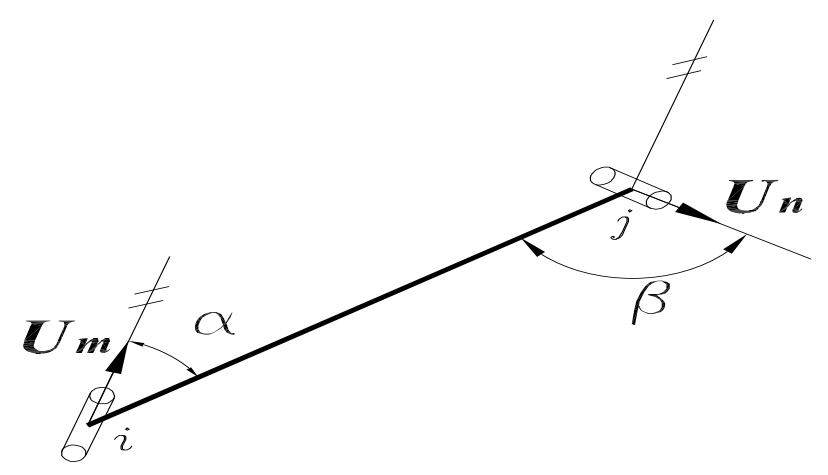

Figure 4: Link with two basic points and two unit vectors

\subsection{Scissor like element}

A scissor like element (SLE) or pantograph is shown in the Figure 1. The link $i j$ and $k l$ are coplanar and can rotate around the pivot. It is assumed that the two links are not equal and pivot is not in the middle. 
The position vectors must satisfy

$$
\frac{b}{a+b} \mathbf{P}_{i}+\frac{a}{a+b} \mathbf{P}_{j}-\frac{c}{c+d} \mathbf{P}_{l}-\frac{d}{c+d} \mathbf{P}_{k}=0
$$

where, $\mathbf{P}_{m}(m=i, j, k, l)$ represents the position vector consisting of coordinates of basic points, and the lengths $a, b, c$ and $d$ are as shown in Figure 1.

\subsection{Boundary constraints}

Boundary constraints are used to exclude the global motion of the mechanism. If the basic point $p$ is fixed, its Cartesian coordinates are zero. If point $q$ moves along a plane perpendicular to $Z$ axis, its $Z$ coordinate is zero. These equations are written as

$$
X_{p}=0=Y_{p}=Z_{p}=Z_{q}
$$

\subsection{System constraint equations}

The rigid constraint equations, joint constraints and boundary constraints in a mechanism can be written together as

$$
f_{j}\left(X_{1}, X_{2}, X_{3}, \cdots, X_{n}\right)=0 \text { for } j=1 \text { to } n_{c}
$$

where $n_{c}$ represents the total number of constraint equations including rigid link, all joint constraints and boundary constraints, and $n$ is the number of Cartesian coordinates of the system. The derivative of the constraint equations, with respect to time give the Jacobian matrix, can be symbolically written as

$$
[\mathbf{B}] \dot{\mathbf{X}}=\mathbf{0}
$$

Since, equation (6) is homogeneous, one can obtain a non-null $\dot{\mathbf{X}}$ if the dimension of the null-space of $[\mathbf{B}]_{n_{c} \times n}$ is at least one. The existence of the null-space implies that the mechanism possess a degree-of-freedom along the corresponding $\dot{\mathbf{X}}$ [5]. The null space of [B] can be obtained numerically or symbolically. A numerical algorithm can only give the degreeof-freedom at a chosen configuration and this is presented next. A symbolic computation of the null space can lead to obtaining the global degree-of-freedom, and this is discussed in Section 5 .

\subsection{Numerical Algorithm}

For a deployment mechanism with $p$ basic points, the number of Cartesian coordinates is $3 p$ and, in addition, the mechanism can have $n_{l}$ length constraints. The mechanism at this stage will have only spherical joints at basic points (for a spatial system) and revolute joints at basic points (for the planar system). The main steps in the numerical algorithm are: 
1. Add the derivative of the constraint equations one at a time in the following order

- arising out of length constraints

- arising out of SLE constraints

2. At each step the dimension of null space of $[\mathbf{B}]$ is evaluated. If dimension of null space of $[\mathbf{B}]$ doesn't decrease when a constraint is added it is redundant.

3. Boundary constraints are added last and the dimension of null space of $[\mathbf{B}]$ is evaluated. If dimension of null space does not decrease after adding a boundary constraint, then corresponding constraint is redundant.

4. The final dimension of the null space of $[\mathbf{B}]$ is the degree-of-freedom of the system.

In choosing the basic points the finite dimensions of joints are not taken into account. In this formulation the initial folded configuration is not considered as it can have many singular configurations and hence does not give the true degrees-of-freedom of the system. The numerical values of basic points at an intermediate configuration are taken for the evaluation of null space of the Jacobian matrix.

\section{Closed form solution for triangular mast}

The numerical algorithm presented above does not give the closed-form solutions for direct and inverse kinematics of masts. To get closed-form solutions, it is useful to get a minimal set of constraint equations and these can be obtained from the original constraint equations by eliminating the unwanted variables. Elimination of variables from a set of nonlinear equations is known to be an extremely hard problem and the difficulty increases with the number and complexity of each equation in the set. Symbolic computation software, MATHEMATICA, has been used to obtain closed-form solutions for some masts. The natural coordinates are useful in this respect since the equations are at most quadratic in the variables used. In this section, the approach to obtain the closed-form solution for a triangular and box mast with spherical and revolute joints is presented.

\subsection{Triangular mast with spherical joints}

For the triangular mast, shown in Figure 2, the assumptions are

1. the links of SLE's are equal in length and the pivot for the SLE is at the midpoint of the links,

2. the joints 1 to 6 are spherical joints and the joints 2 and 3 are constrained to move in a plane,

3. the joint 1 is fixed, 
4. the links are rigid and the cables used for pre-stressing does not affect the kinematics.

Using equation (1), the length constraint equation for link 1-5 is given by

$$
\left(X_{5}-X_{1}\right)^{2}+\left(Y_{5}-Y_{1}\right)^{2}+\left(Z_{5}-Z_{1}\right)^{2}-L_{15}^{2}=0
$$

and additional five similar equations for the other links can be obtained. This leads to six length constraint equations for the triangular mast.

The SLE equations can be obtained by using equation (3) as

$$
\begin{aligned}
& \mathbf{P}_{1}+\mathbf{P}_{5}-\mathbf{P}_{4}-\mathbf{P}_{2}=0 \\
& \mathbf{P}_{2}+\mathbf{P}_{6}-\mathbf{P}_{3}-\mathbf{P}_{5}=0 \\
& \mathbf{P}_{3}+\mathbf{P}_{4}-\mathbf{P}_{1}-\mathbf{P}_{6}=0
\end{aligned}
$$

It can be observed that only two of the three SLE equations are independent. The $Z$ component of the above equations and the use of assumptions 2 and 3, results in

$$
Z_{4}=Z_{5}=Z_{6}=Z
$$

Now using the fact that joint 1 is fixed gives $X_{1}=Y_{1}=Z_{1}=0$. Using this in the six length constraints and equations (8), after simplification, $X_{4}$ and $Y_{4}$ are obtained to be 0 . Substituting $X_{4}=Y_{4}=0$ and after simplification, three independent equations with five variables are obtained. These are given as

$$
\begin{gathered}
X_{2}{ }^{2}+Y_{2}{ }^{2}+Z^{2}-L^{2}=0 \\
\left(X_{3}-X_{2}\right)^{2}+\left(Y_{3}-Y_{2}\right)^{2}+Z^{2}-L^{2}=0 \\
X_{3}{ }^{2}+Y_{3}{ }^{2}+Z^{2}-L^{2}=0
\end{gathered}
$$

where $Z$ is defined in equation (9) and all $L_{i j}$ 's are equal to $L$ from assumption 1.

Assuming $X_{2}, Y_{2}$ as known inputs, the solution for $X_{3}, Y_{3}$, and $Z$ are given as

$$
\begin{aligned}
Z & = \pm \sqrt{L^{2}-\left(X_{2}^{2}+Y_{2}^{2}\right)} \\
X_{3} & =\frac{1}{2}\left(X_{2} \mp \sqrt{3} Y_{2}\right) \\
Y_{3} & =\frac{1}{2}\left(Y_{2} \pm \sqrt{3} X_{2}\right)
\end{aligned}
$$

From equations (13) and (8), the coordinates of all the joints of the triangular mast can be obtained in closed form. It can be observed from the equations (13) that for the given $X_{2}, Y_{2}$ coordinates of joint 2 , four configurations of masts are possible - two configurations each for the positive and negative $Z$ coordinate, respectively. Each configuration is the mirror image of the triangle formed about the line joining the joints 2 and 1 . If the mast moves only along the positive $Z$ axis, the number of kinematic solution the mast can have is 2 . 
From equations (13), $L^{2}-\left(X_{2}{ }^{2}+Y_{2}^{2}\right) \geq 0$. If $L^{2}-\left(X_{2}{ }^{2}+Y_{2}^{2}\right)=0$, the coordinates of joint 2 lie in a circle of radius $L$. This corresponds to the fully deployed configuration with $Z=0$. Depending on the magnitude of coordinates the joint 2 moves along the circle of radius $L$. If $L^{2}-\left(X_{2}^{2}+Y_{2}^{2}\right)<0$, the solution for $Z$ is imaginary and the coordinates of joint 2 lie out side the workspace of the mast. The condition $L^{2}-\left(X_{2}{ }^{2}+Y_{2}{ }^{2}\right)>0$, covers the entire range of motion from the fully folded $(Z=L)$ to very close to the fully deployed configuration $(Z \approx 0)$.

In the above analysis the independent variables are taken as the coordinates of joint 2 . The above method can be used by taking the coordinates of joint 3 as independent variables. Alternatively equations (13) containing $X_{3}$ and $Y_{3}$, can be simultaneously solved to evaluate the coordinates $X_{2}$ and $Y_{2}$ in terms of $X_{3}$ and $Y_{3}$ and equation (12) can be used to evaluate the coordinate of $Z$.

\subsection{Box mast with spherical joints}

For the box mast shown in figure 3, there are eight length constraint equations and three independent SLE equations. Using an approach similar to the triangular mast, the sets of closed-form solutions for the box mast are obtained. The sets of closed-form solutions with $X_{2}, Y_{2}$ and $X_{3}$ as input, and for positive $Z$, are given below.

\section{Solution 1}

$$
\begin{aligned}
Y_{3} & =Y_{2}+P \\
X_{4} & =X_{2} \\
Y_{4} & =Y_{2} \\
Z & =\sqrt{L^{2}-\left(X_{2}^{2}+Y_{2}^{2}\right)}
\end{aligned}
$$

Solution 2

$$
\begin{aligned}
Y_{3} & =Y_{2}-P \\
X_{4} & =X_{2} \\
Y_{4} & =Y_{2} \\
Z & =\sqrt{L^{2}-\left(X_{2}^{2}+Y_{2}^{2}\right)}
\end{aligned}
$$

\section{Solution 3}

$$
\begin{aligned}
Y_{3} & =Y_{2}+P \\
X_{4} & =-X_{2}+X_{3} \\
Y_{4} & =P \\
Z & =\sqrt{L^{2}-\left(X_{2}^{2}+Y_{2}^{2}\right)}
\end{aligned}
$$

\section{Solution 4}

$$
Y_{3}=Y_{2}-P
$$




$$
\begin{aligned}
X_{4} & =-X_{2}+X_{3} \\
Y_{4} & =-P \\
Z & =\sqrt{L^{2}-\left(X_{2}^{2}+Y_{2}^{2}\right)}
\end{aligned}
$$

where $P=\sqrt{2 X_{2} X_{3}-X_{3}^{2}+Y_{2}^{2}}$ in all the above solutions.

The box mast has eight solutions - four each for positive and negative $Z$. Each configuration has two folded type with SLE's in one line and two deployed type with SLE's separated by a known angle. In the $Z$ coordinate equation, $L^{2}-\left(X_{2}{ }^{2}+Y_{2}^{2}\right) \geq 0$. Again the observations are similar to the triangular mast - 1) If $L^{2}-\left(X_{2}^{2}+Y_{2}^{2}\right)=0$, the coordinates of joint 2 lie in a circle of radius $L$, 2) If $L^{2}-\left(X_{2}{ }^{2}+Y_{2}^{2}\right)<0$, the solution for $Z$ are imaginary and the coordinates of joint 2 lie out side the work space of the mast, and 3 ) for $L^{2}-\left(X_{2}^{2}+Y_{2}^{2}\right)>0$, all the mast configuration (except $Z=0$ ) are covered.

Another set of observations come from the equation for $P$. If $P=0$, then $X_{3}=X_{2} \pm$ $\sqrt{\left(X_{2}^{2}+Y_{2}^{2}\right)}$, which leads to $X_{3}=X_{2} \pm L$. For the positive sign, the mast has two solutions. In the first solution, the first and fourth SLE are collinear and lie along the circle of radius $L$, and the second and third SLE are collinear and horizontal. In the second solution the mast is like a parallelogram. For $P>0$, four solutions of the mast are possible. The first two solutions show co-linearity of SLE's and the last two solutions show separation of SLEs at a known angle. Again if $P<0$, the roots are imaginary the mast cannot reach these configurations.

\subsection{Triangular masts with revolute joints}

For the triangular mast shown in Figure 2, the assumptions described in the previous section are used except that each spherical joint is replaced by the two revolute joints whose axes is normal to the plane of the SLE (see Figure 5).

The revolute joint constraint for the face 1-2-5-4 of triangular mast is obtained by using any one of equations (2), and are given by

$$
\begin{aligned}
& \left(X_{2}-X_{4}\right) n_{1_{x}}+\left(Y_{2}-Y_{4}\right) n_{1_{y}}=0 \\
& \left(X_{1}-X_{5}\right) n_{1_{x}}+\left(Y_{1}-Y_{5}\right) n_{1_{y}}=0
\end{aligned}
$$

Similarly, the revolute joint constraints for the face 2-3-6-5 is given by

$$
\begin{aligned}
& \left(X_{2}-X_{6}\right) n_{2_{x}}+\left(Y_{2}-Y_{6}\right) n_{2_{y}}=0 \\
& \left(X_{3}-X_{5}\right) n_{2_{x}}+\left(Y_{3}-Y_{5}\right) n_{2_{y}}=0
\end{aligned}
$$

The revolute joint constraint for the face 1-3-6-4 are given by

$$
Y_{1}-Y_{6}=0, Y_{3}-Y_{4}=0
$$




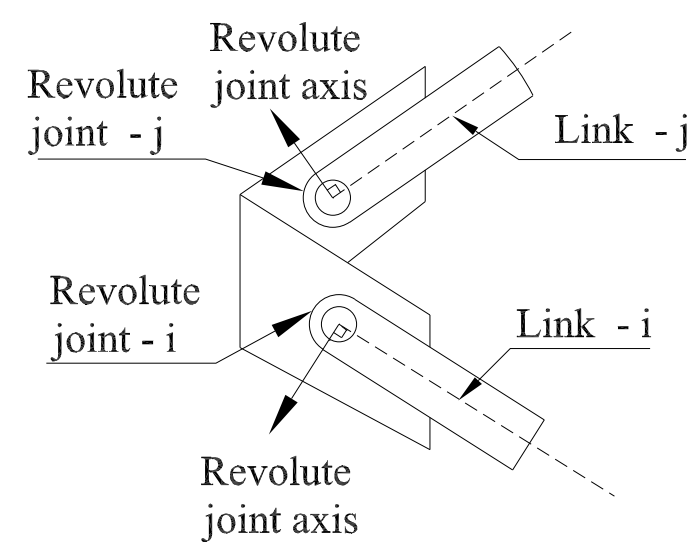

Figure 5: A spherical joint replaced by two revolute joints

where for the three SLE's, $U_{m}$ or $U_{n}$ is $\left\{n_{1_{x}}, n_{1_{y}}, 0\right\}^{T},\left\{n_{2_{x}}, n_{2_{y}}, 0\right\}^{T},\{0,1,0\}^{T}$.

Using the revolute joint constraints of face 1-3-6-4, and substituting in SLE equations (8), results in

$$
Y_{3}+Y_{4}=0, \quad Y_{3}-Y_{4}=0
$$

which give $Y_{3}=0$ and $Y_{4}=0$ as solutions. Substituting SLE equations in length constraint equations and substituting equations (17), gives

$$
\begin{aligned}
\left(X_{2}+X_{4}\right)^{2}+Y_{2}^{2}+Z^{2}-L^{2} & =0 \\
\left(X_{2}-X_{4}\right)^{2}+Y_{2}^{2}+Z^{2}-L^{2} & =0 \\
\left(X_{2}-X_{3}-X_{4}\right)^{2}+Y_{2}^{2}+Z^{2}-L^{2} & =0 \\
\left(-X_{2}+X_{3}-X_{4}\right)^{2}+Y_{2}^{2}+Z^{2}-L^{2} & =0 \\
\left(X_{3}+X_{4}\right)^{2}+Z^{2}-L^{2} & =0 \\
\left(X_{3}-X_{4}\right)^{2}+Z^{2}-L^{2} & =0
\end{aligned}
$$

Using the first and the fourth equations in equation (18), and on expanding and simplifying yields

$$
X_{3}=0, X_{3}=2\left(X_{2}+X_{4}\right)
$$

For $X_{3}=0$, simplification results in $X_{4}=0$ and $Z= \pm \sqrt{L}$ which in turn leads to $X_{2}=Y_{2}=0$. This represents the fully folded or stowed configuration of the mast.

For $X_{3}=2\left(X_{2}+X_{4}\right)$, simplification results in

$$
X_{4}=0
$$




$$
\begin{aligned}
X_{3} & =2 X_{2} \\
Z & = \pm \sqrt{L^{2}-4 X_{2}^{2}} \\
Y_{2} & = \pm \sqrt{3} X_{2}
\end{aligned}
$$

The expressions above relate all the coordinates as a function of a given $X_{2}$ implying a single degree-of-freedom system. The triangular mast can have single degree-of-freedom with only two SLEs and only revolute joints on face 1-3-6-4 and spherical joints at other faces.

If instead of the above, the revolute joint constraints equations of face 1-2-5-4 as a function of the direction cosines are used, one can get exactly the same expressions as in equation (20).

\subsection{Box mast with revolute joints}

For the box mast shown in figure 3, the assumptions described in the previous section are used except that each spherical joint is replaced by the two revolute joints each normal to the sides of the mast. The revolute joint constraint for the face 1-2-6-5 of box mast is obtained by using equation (2):

$$
Y_{2}-Y_{5}=0, \quad Y_{1}-Y_{6}=0
$$

The revolute joint constraint for the face 2-3-7-6 is given by

$$
X_{2}-X_{7}=0, X_{3}-X_{6}=0
$$

and similarly, the revolute joint constraint for the other two faces can be obtained.

Using the revolute joint constraints of face 1-2-6-5, and substituting in SLE equation (3) for each face results in

$$
Y_{2}+Y_{5}=0, Y_{2}-Y_{5}=0
$$

which imply that $Y_{2}=0$ and $Y_{5}=0$. Substituting SLE equations in length constraint equations and using the above result, 8 equations similar to equations (18) are obtained. From these one can show that

$$
X_{2}=0 \quad \text { or } \quad X_{5}=0
$$

Since $X_{2}$ is considered to be input $X_{5}=0$. For this case, the eight equations further reduce to four independent equations

$$
\begin{aligned}
X_{2}^{2}+Z^{2}-L^{2} & =0 \\
\left(X_{2}-X_{3}\right)^{2}+Y_{3}^{2}+Z^{2}-L^{2} & =0 \\
X_{4}^{2}+Y_{4}^{2}+Z^{2}-L^{2} & =0 \\
\left(X_{3}-X_{4}\right)^{2}+\left(Y_{3}-Y_{4}\right)^{2}+Z^{2}-L^{2} & =0
\end{aligned}
$$

where $Z_{5}=Z_{6}=Z_{7}=Z_{8}=Z$ and all link lengths $L_{i j}$ 's are equal to $L$. 
The above four equations can be solved to obtain four independent sets of solutions. The four sets are given below:

\section{Solution 1}

$$
\begin{aligned}
Y_{3} & =\sqrt{X_{3}\left(2 X_{2}-X_{3}\right)} \\
X_{4} & =X_{2} \\
Y_{4} & =0 \\
Z & =\sqrt{L^{2}-X_{2}^{2}}
\end{aligned}
$$

\section{Solution 2}

$$
\begin{aligned}
Y_{3} & =-\sqrt{X_{3}\left(2 X_{2}-X_{3}\right)} \\
X_{4} & =X_{2} \\
Y_{4} & =0 \\
Z & =\sqrt{L^{2}-X_{2}^{2}}
\end{aligned}
$$

\section{Solution 3}

$$
\begin{aligned}
Y_{3} & =\sqrt{X_{3}\left(2 X_{2}-X_{3}\right)} \\
X_{4} & =-X_{2}+X_{3} \\
Y_{4} & =\sqrt{X_{3}\left(2 X_{2}-X_{3}\right)} \\
Z & =\sqrt{L^{2}-X_{2}^{2}}
\end{aligned}
$$

\section{Solution 4}

$$
\begin{aligned}
Y_{3} & =-\sqrt{X_{3}\left(2 X_{2}-X_{3}\right)} \\
X_{4} & =-X_{2}+X_{3} \\
Y_{4} & =-\sqrt{X_{3}\left(2 X_{2}-X_{3}\right)} \\
Z & =\sqrt{L^{2}-X_{2}^{2}}
\end{aligned}
$$

It can be observed from the above solutions that the box mast with revolute joints has apparently two degree-of-freedom system, as it needs two inputs to specify its configuration. Hence, the box mast can have two degree-of-freedom with only three SLEs and revolute joints on one face and spherical joints at other faces. However, using the revolute joint constraints of face 2-3-7-6, and following a similar procedure, a single degree-of-freedom mechanism can be obtained. The expressions for the dependent variables in terms of the input $X_{2}$ are

$$
\begin{aligned}
Z & = \pm \sqrt{L^{2}-X_{2}^{2}} \\
Y_{2} & =0
\end{aligned}
$$




$$
\begin{aligned}
X_{3} & =X_{2} \\
Y_{3} & = \pm X_{2} \\
X_{4} & =0 \\
Y_{4} & = \pm X_{2}
\end{aligned}
$$

The box mast can have single degree-of-freedom with three SLEs and only revolute joints on the two faces and spherical joints at other faces.

\section{Global DOF evaluation through null space}

The evaluation of degrees-of-freedom using null space of Jacobian matrix is at a given configuration and does not give the degrees-of-freedom in the entire domain. In the stowed configuration/intermediate configuration, the links and joint axis can align resulting in some elements of the Jacobian matrix becoming zero leading higher degrees-of-freedom. To evaluate the degrees-of-freedom in the entire domain, symbolical evaluation of the null space of the $[\mathbf{B}]$ matrix is performed and the closed-form equations derived in section 4 are used. This is illustrated for the triangular and the box masts.

\subsection{Triangular mast}

The Jacobian matrix with length constraint equations, the SLE equations and boundary constraints is given by

$$
\left[\mathbf{B}_{p_{1}}\left(X_{1}, \cdots X_{6}, Y_{1}, \cdots Y_{6}, Z\right)\right] \dot{\mathbf{X}}_{p_{1}}=0
$$

where, $\mathbf{X}_{p_{1}}=\left\{X_{1}, \cdots X_{6}, Y_{1}, \cdots Y_{6}, Z\right\}^{T}$. The dependent variables in the above equation can be eliminated by using the closed-form solutions given in equations (13) for $Z, X_{3}$ and $Y_{3}$. On simplification, one can get

$$
\left[\mathbf{B}_{p_{2}}\left(X_{2}, Y_{2}\right)\right] \dot{\mathbf{X}}_{p_{2}}=0
$$

where $\mathbf{X}_{p_{2}}$ is the vector $\left\{X_{2}, Y_{2}\right\}^{T}$ and the matrix is a function of $X_{2}$ and $Y_{2}$ alone. The null space of the matrix can now be more easily evaluated symbolically in MATHEMATICA. The dimension of null space gives the degree-of-freedom of the system in entire workspace except at origin. The dimension of the null space was found to be two and this is consistent with the closed-form solutions obtained for the mast with spherical joints.

An alternate method is to use the three equations (10)- (12). By taking the derivative of these three equations, the Jacobian matrix, can be symbolically evaluated as

$$
\left[\mathbf{B}_{p_{3}}\left(X_{2}, Y_{2}, X_{3}, Y_{3}, Z\right)\right] \dot{\mathbf{X}}_{p_{3}}=0
$$

where $\mathbf{X}_{p_{3}}$ is the vector $\left\{X_{2}, Y_{2}, X_{3}, Y_{3}, Z\right\}^{T}$. The variables $X_{3}, Y_{3}$ and $Z$ can be eliminated, to get 


$$
\left[\mathbf{B}_{p_{4}}\left(X_{2}, Y_{2}\right)\right] \dot{\mathbf{X}}_{p_{2}}=0
$$

The null space of the matrix obtained in this way was also found to be two.

\subsection{Box mast}

Similar to the triangular mast, using the length constraint equations, simplifying and substituting four solutions of the box mast given in section 4.2 for $Y_{3}, X_{4}, Y_{4}$ and $Z$, leads to

$$
\left[\mathbf{B}_{p}\left(X_{2}, Y_{2}, X_{3}\right)\right] \dot{\mathbf{X}}_{p}=0
$$

where the matrix $\left[\mathbf{B}_{p}\right]$ is a function of $X_{2}, Y_{2}$ and $X_{3}$, and $\mathbf{X}_{p}$ is the vector $\left\{X_{2}, Y_{2}, X_{3}\right\}^{T}$. The null space is evaluated for this matrix symbolically and the dimension of null space gives the degrees-of-freedom of the box mast. The degree-of-freedom, except at origin, was obtained to be three and this again agrees with the results obtained in section 4.2.

\section{Results and discussions}

In this section, the results obtained using the numerical algorithm described in section 3.6 and kinematic simulation of a single triangular mast using the closed-form solutions are presented. For the triangular and box mast, the redundant joints are obtained.

\subsection{Degree-of-freedom and redundancy evaluation}

The triangular mast shown in figure 2 has six rigid link constraints, 3 SLE constraints and fixed boundary conditions at joint 1 . The additional boundary conditions at points 2 and 3 are required to ensure the motion in $X-Y$ plane. The results of null space are presented in Table 1. It is observed from the Table 1 that the null space reduces by three by adding the first SLE. The null space reduces by only 2 for the $2^{\text {nd }}$ SLE. The null space does not change for the $3^{\text {rd }}$ SLE. The dimension of null space for the triangular mast is 2 .

The results of null space for box mast shown in figure 3 are presented in Table 2. It is observed from the table that the null space reduces by three by adding each SLE's. The null space reduces by only 2 for the $3^{\text {rd }}$ SLE. The null space does not change for the $4^{\text {th }}$ SLE. The dimension of null space for the box masts is 3 . It is observed from the last row that the rank does not increase by adding additional boundary conditions because three boundary conditions are sufficient to represent the motion in a plane.

Similar analysis was also carried out for the hexagonal mast and similar behavior was observed. Hence, for a $n$-sided SLE mast, null space reduces by three for addition of each SLE and the null space reduces by only 2 for addition of $(n-1)^{\text {th }}$ SLE. The null space does not change for the addition of the $n^{\text {th }}$ SLE. Hence the last SLE in a $n$ sided SLE based mast is redundant. 


\begin{tabular}{|c|c|c|c|}
\hline Constraints & Size of $[\mathbf{B}]$ & Null Space & Remarks \\
\hline Length constraints & $(6,18)$ & 12 & \\
\hline + SLE 1 & $(9,18)$ & 9 & \\
\hline + SLE 2 & $(12,18)$ & 7 & One component redundant \\
\hline + SLE 3 & $(15,18)$ & 7 & SLE - 3 redundant \\
\hline $\begin{array}{c}\text { + Boundary conditions } \\
\left(X_{1}=Y_{1}=Z_{1}=0\right)\end{array}$ & $(18,18)$ & 4 & \\
\hline $\begin{array}{c}\text { Boundary conditions } \\
\left(Z_{2}=Z_{3}=0\right)\end{array}$ & $(20,18)$ & 2 & \\
\hline
\end{tabular}

Table 1: [B] Matrix details for triangular mast

\begin{tabular}{|c|c|c|c|}
\hline Constraints & Size of $[\mathbf{B}]$ & Null Space & Remarks \\
\hline Length constraints & $(8,24)$ & 16 & \\
\hline+ SLE 1 & $(11,24)$ & 13 & \\
\hline+ SLE 2 & $(14,24)$ & 10 & \\
\hline+ SLE 3 & $(17,24)$ & 8 & One component redundant \\
\hline+ SLE 4 & $(20,24)$ & 8 & SLE - 4 redundant \\
\hline $\begin{array}{c}\text { + Boundary conditions } \\
\left(X_{1}=Y_{1}=Z_{1}=0\right)\end{array}$ & $(23,24)$ & 5 & \\
\hline $\begin{array}{c}\text { Boundary conditions } \\
\left(Z_{2}=Z_{3}=Z_{4}=0\right)\end{array}$ & $(26,24)$ & 3 & One of $Z$ 's redundant \\
\hline
\end{tabular}

Table 2: [B] Matrix details for box mast

\subsection{Mast with revolute joints}

Each spherical joints of masts shown in figure 2 and figure 3 are replaced by two revolute joints (see figure 5). The revolute joint constraints of each face is taken to evaluate the null space.

\subsubsection{Case - 1}

In the first approach the revolute joints are added for each face and SLEs are added subsequently. The null space is evaluated for each addition. The results for triangular and box mast are presented in Table 3 and Table 4 respectively. It is observed from the table that the last two SLEs are redundant for both the masts. Hence, with all revolute joints only one SLE is sufficient to achieve single degree-of-freedom. The analysis has also been carried out for the hexagonal mast. It was observed that the last three SLE,s are redundant. Hence, three SLE,s are sufficient to get the single degree-of-freedom for the hexagonal mast. 


\begin{tabular}{|c|c|c|c|}
\hline Constraints & Size of $[\mathbf{B}]$ & Null Space & Remarks \\
\hline Length constraints & $(6,18)$ & 12 & \\
\hline Revolute joints & & & \\
\hline + FACE 1 & $(8,18)$ & 10 & \\
\hline + FACE 2 & $(10,18)$ & 8 & \\
\hline + FACE 3 & $(12,18)$ & 6 & \\
\hline SLEs & & & \\
\hline + SLE 1 & $(15,18)$ & 4 & \\
\hline + SLE 2 & $(18,18)$ & 4 & SLE -2 is redundant \\
\hline + SLE 3 & $(21,18)$ & 4 & SLE - 3 is redundant \\
\hline $\begin{array}{c}\text { Boundary conditions } \\
X_{1}=Y_{1}=Z_{1}=0\end{array}$ & $(24,18)$ & 1 & \\
\hline
\end{tabular}

Table 3: [B] Matrix details for triangular mast

\begin{tabular}{|c|c|c|c|}
\hline Constraints & Size of $[\mathbf{B}]$ & Null Space & Remarks \\
\hline Length constraints & $(8,24)$ & 16 & \\
\hline Revolute joints & & & \\
\hline+ FACE 1 & $(10,24)$ & 14 & \\
\hline+ FACE 2 & $(12,24)$ & 12 & \\
\hline+ FACE 3 & $(14,24)$ & 10 & \\
\hline+ FACE 4 & $(16,24)$ & 8 & \\
\hline SLEs & & & \\
\hline+ SLE 1 & $(19,24)$ & 5 & two components are redundant \\
\hline+ SLE 2 & $(22,24)$ & 4 & SLE - 3 is redundant \\
\hline+ SLE 3 & $(25,24)$ & 4 & SLE - 4 is redundant \\
\hline+ SLE 4 & $(28,24)$ & 4 & \\
\hline+ Boundary conditions & & & \\
$X_{1}=Y_{1}=Z_{1}=0$ & $(31,24)$ & 1 & \\
\hline
\end{tabular}

Table 4: [B] Matrix details for box mast

\subsubsection{Case -2}

In the second approach the SLEs are introduced first and revolute joints are introduced later and the dimension of null space is evaluated at each stage. The results are presented in Table 5 and Table 6 . It is observed that the last SLE is redundant for both the masts. It is also observed that the revolute joints for the $3^{\text {rd }}$ face for the triangular mast and $3^{\text {rd }}$ and $4^{\text {th }}$ face are redundant for the box masts. Hence, these faces can have spherical joints with out changing the degree-of-freedom.

From the tables, it can be observed that when the revolute joints are added first and SLE's 
added later, the last two SLE's were redundant. By reversing the order of the introduction of joints and SLE's, it was observed that the last SLE was redundant and the revolute joints on the last face for the triangular mast and the revolute joints on the last two faces for box mast were redundant. However, the order of adding the constraints does not change the final degree-of-freedom.

\begin{tabular}{|c|c|c|c|}
\hline Constraints & Size of $[\mathbf{B}]$ & Null Space & Remarks \\
\hline Length constraints & $(6,18)$ & 12 & \\
\hline SLEs & & & \\
\hline + SLE 1 & $(9,18)$ & 9 & \\
\hline + SLE 2 & $(12,18)$ & 7 & SLE - 3 is redundant \\
\hline + SLE 3 & $(15,18)$ & 7 & \\
\hline Revolute joints & & & one component is redundant \\
\hline + FACE 1 & $(15,18)$ & 5 & Joints on FACE 3 are redundant \\
\hline + FACE 2 & $(18,18)$ & 4 & \\
\hline+ FACE 3 & $(21,18)$ & 4 & \\
\hline $\begin{array}{c}\text { Boundary conditions } \\
X_{1}=Y_{1}=Z_{1}=0\end{array}$ & $(24,18)$ & 1 & \\
\hline
\end{tabular}

Table 5: [B] Matrix details for triangular mast

\begin{tabular}{|c|c|c|c|}
\hline Constraints & Size of $[\mathbf{B}]$ & Null Space & Remarks \\
\hline Length constraints & $(8,24)$ & 16 & \\
\hline SLEs & & & \\
\hline + SLE 1 & $(11,24)$ & 13 & \\
\hline+ SLE 2 & $(14,24)$ & 10 & \\
\hline+ SLE 3 & $(17,24)$ & 8 & SLE - 4 is redundant \\
\hline + SLE 4 & $(20,24)$ & 8 & \\
\hline Revolute joints & & & \\
\hline+ FACE 1 & $(22,24)$ & 6 & \\
\hline+ FACE 2 & $(24,24)$ & 4 & Joints on FACE 3 are redundant \\
\hline+ FACE 3 & $(26,24)$ & 4 & Joints on FACE 4 are redundant \\
\hline+ FACE 4 & $(28,24)$ & 4 & \\
\hline+ Boundary conditions & & & \\
$X_{1}=Y_{1}=Z_{1}=0$ & $(31,24)$ & 1 & \\
\hline
\end{tabular}

Table 6: [B] Matrix details for Box mast 


\subsection{Kinematic simulation for the triangular mast}

The kinematic simulations for a triangular mast using the closed-form results obtained in section 4.3 was performed. The triangular mast in a fully stowed configuration is taken as the initial configuration. The coordinate $X_{2}$ is varied from 0 to 30 in steps of 2 and $L=30.0$. Equation 20 is solved to obtain the coordinates of joint 3 as the mast deploys. The simulation is shown in Figure 6. It is observed from the Figure 6 that as the joint 3 moves horizontally, the two solutions of joint 2 moves along the $+60^{\circ}$ and $-60^{\circ}$ line about $X$ axis. The decrease in height of the mast, as shown by the $Z$ coordinates of joints 4,5 and 6 , during deployment is also shown.
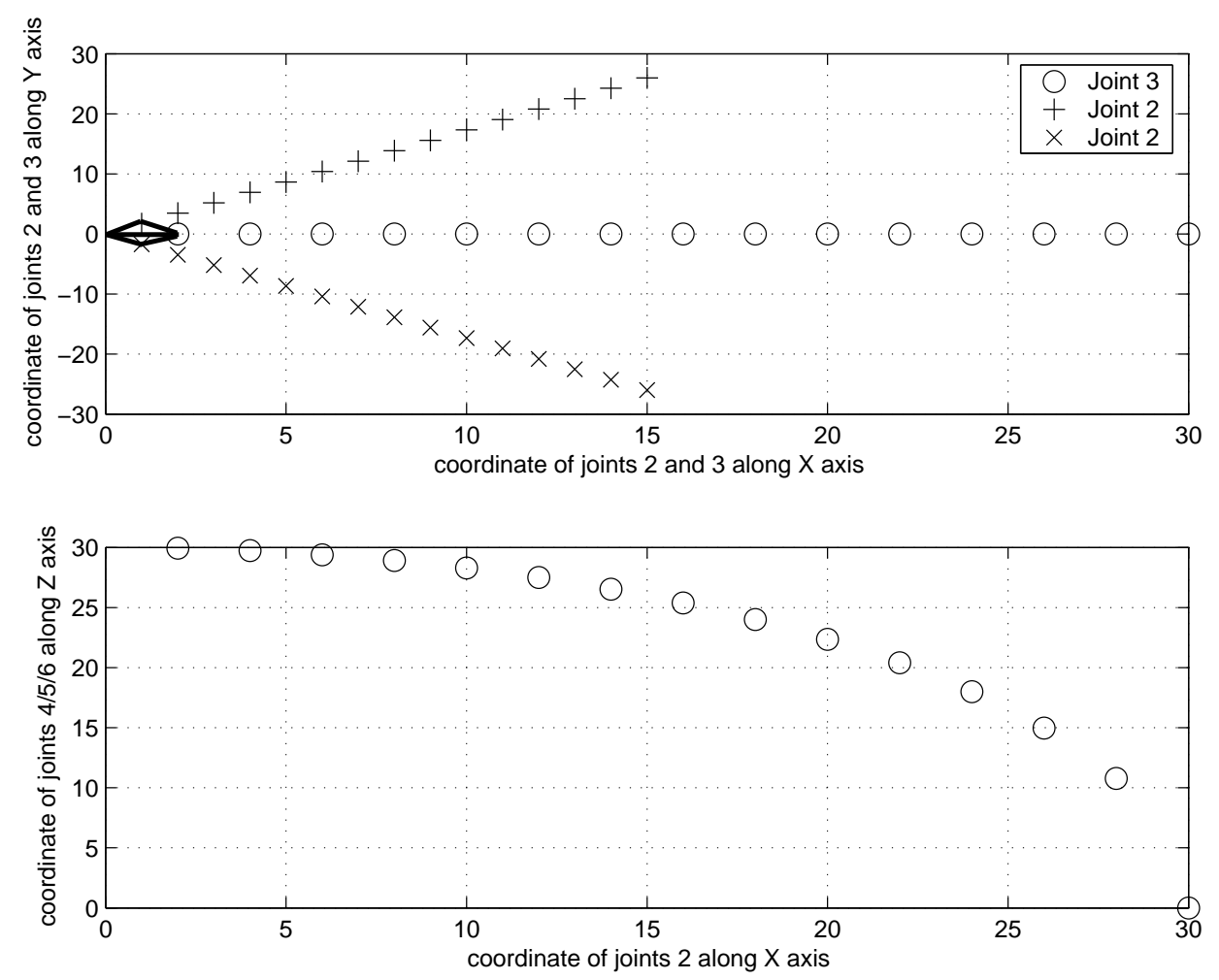

Figure 6: Trajectory of joint coordinates for triangular mast

\section{Conclusions}

In this paper the Cartesian coordinate approach has been used to obtain the kinematic equations for the three dimensional deployable SLE masts. The degree-of-freedom of the masts were evaluated from the dimension of null space of the Jacobian matrix formed by the derivative of the constraint equations. An algorithm was presented to identify the redundant kinematic pairs in the mechanism. For masts with spherical joints, it was observed that the last SLE is redundant for the triangular and box mast, and boundary conditions were 
redundant as the number of sides of the mast is increased. Hence, these masts can achieve the required degree-of-freedom without these kinematic pairs. The order of adding constraints, for masts with revolute joints, does not change the final obtained degree-of-freedom.

The kinematics of triangular and box masts were studied symbolically for mast with both spherical and revolute joints. The closed-form results confirmed the redundancy in SLEs and revolute joints. The symbolic approach was used to obtain the global degree-of-freedom and results were obtained for the triangular and box mast with spherical joints.

Finally, the kinematic simulation results for a triangular mast with revolute joints, using the closed-form results, have been presented. The theory and algorithms presented in this paper can be extended to masts of different shapes and for the stacked masts.

\section{References}

[1] A. K. S. Kwan and S. Pellegrino, "A cable rigidized 3D pantograph," Proc. of 4rth European symposium on Mechanisms and Tribology, France, Sept. 1989 (ESA-SP-299, March 1990).

[2] Z. You and S. Pellegrino, "Cable stiffened pantographic deployable structures, Part1: Triangular mast," AIAA Journal, Vol. 34, No. 4, pp. 813-820, 1996.

[3] A. K. S. Kwan and S. Pellegrino, "Active and passive elements in deployable / retractable masts," International Journal of Space Structures, Vol. 8, No. 1 and 2, pp. 29-40, 1993

[4] R. S. Hartenberg and J. Denavit, Kinematic synthesis of linkages, McGraw Hill, 1965.

[5] J. Garcia De Jalon and E. Bayo, Kinematic and dynamic simulation of multi-body systems: The real time challenge, Springer Verlag, 1994.

[6] J. Garcia De Jalon and Migual Angel Serna, "Computer methods for kinematic analysis of lower pair mechanism -1 Velocities and acceleration," Mechanism and Machine Theory, Vol. 17, No 6, pp. 397-403, 1982.

[7] J. Garcia De Jalon, J. Unda and A. Avello, "Natural coordinates of computer analysis of multi body systems," Computer Methods in Applied Mechanics and Engineering, Vol. 56, pp. 309-327, 1986.

[8] T. Langbecker, "Kinematic analysis of deployable scissor structures," International Journal of Space Structures, Vol. 14, No. 1, pp. 1-15, 1999.

[9] W. J. Chen, G. Y. Fu, J. H. Gohg, Y. L. Hi and S. L. Dong, "Dynamic deployment simulation for pantographic deployable masts," Mechanics of Structures and Machines, Vol. 30, pp. 249-277, 2002.

[10] S. Wolfram, Mathematica, Second Edition, Addition Wesley Publishing Co. 1991. 
[11] J. S. Dai, Duanling Li, Qixian Zhang and Guoguang Jin, "Mobility analysis of a complex structured ball based on mechanism decomposition and equivalent screw system analysis," Mechanism and Machine Theory, Vol. 39, pp. 445-458, 2004.

[12] J. S. Dai and J. Rees Jones, "Mobility in metamorphic mechanism of foldable/erectable kinds," Trans. of ASME, Journal of Mechanical Design, Vol. 121, pp. 375-382, 1999.

[13] J. S. Zhao, K. Zhao and Z. J. Feng, "A theory of degrees of freedom for mechanisms," Mechanism and Machine Theory, Vol. 39, No. 6, pp. 621-644, 2004.

[14] G. Gogu, "Mobility of mechanisms : A critical review," Mechanism and Machine Theory, Vol. 40, pp. 1068 -1097, 2005.

[15] L-W. Tsai, Enumeration of kinematic structures according to function, CRC Press, 2001.

[16] D. B. Warnear and M. Chew, "Kinematic synthesis of deployable foldable truss structures using graph theory, Part 1: Graph generation," Trans. of ASME, Journal of Mechanical Design, Vol. 117, pp. 112-116, 1995.

[17] D. B. Warnear and M. Chew, "Kinematic synthesis of deployable foldable truss structures using graph theory Part 2: Generation of deployable truss module design concepts," Trans. of ASME, Journal of Mechanical Design, Vol. 117, pp 117-122, 1995.

[18] F. Freudenstein, "On the verity of motions generated by mechanism," Trans. of ASME, Journal of Engineering for Industry, Vol. 84, pp. 156-160, 1962.

[19] E. N. Kuznetsov, "Under constrained structural systems," International Journal of Solids and Structures, Vol. 24, No. 2, pp. 153-163, 1988. 